\title{
Nicht für immer. Ars moriendi nova - Sterbekunst ohne Jenseitsperspektive
}

Nina Streeck

Sterben ist das Letzte.

- Horaz

Sterben sei eine Kunst suggeriert der seit einigen Jahren vermehrt wieder bemühte Begriff der ars moriendi. ${ }^{1}$ In mehr oder weniger enger Orientierung an der namensgebenden christlichen "Sterbekunst« des Mittelalters wird unter diesem Titel nach gegenwärtig tauglichen Antworten auf die Frage nach dem guten Tod gesucht, oft getragen von dem Anliegen, den Kontext von Entscheidungen am Lebensende zu erhellen und die Dimension des guten Lebens in moralphilosophische Überlegungen einzubeziehen. ${ }^{2}$ Die Notwendigkeit einer ars moriendi nova ergibt sich für deren Verfechterinnen und Verfechter aus dem »Wandel von gesellschaftlichen Vorstellungen in Bezug auf das Lebensende $~^{3}$; das Programm lautet paradigmatisch, die »Bedingungen des gegenwärtigen und absehbar

\footnotetext{
1 Vgl. u.a. Placidus Berger, Ars moriendi. Die Kunst des Lebens und des Sterbens, Münsterschwarzach 2010; Carlo Leget, Retrieving the ars moriendi tradition, in: Medicine, Health Care and Philosophy 10 (2007), 313-319; Simon Peng-Keller/Pierre Bühler (Hg.), Bildhaftes Erleben in Todesnähe: Hermeneutische Erkundungen einer heutigen ars moriendi, Zürich 2014; Daniel Schäfer/Christof Müller-Busch/Andreas Frewer (Hg.), Perspektiven zum Sterben. Auf dem Weg zu einer Ars moriendi nova?, Stuttgart 2012; Jean-Pierre Wils, ars moriendi. Über das Sterben, Frankfurt a.M. 2007. 2 Vgl. etwa Franz Josef Illhardt, Ars moriendi - aktuelle Wiederentdeckung, in: Sterben und Tod. Ein interdisziplinäres Handbuch, hg. v. Héctor Wittwer/Daniel Schäfer/ Andreas Frewer, Stuttgart 2010, 170-174, hier 170: Die Ars-moriendi-Literatur solle "wenigstens allegorisch deutlich machen, dass alle Entscheidungen am Lebensende [...] (1) mit einer lebensweltlichen Kultur zu tun haben, die über die Logik der Entscheidung selbst hinaus geht bzw. ihr vorausliegt, (2) nicht nur und in erster Linie medizinische bzw. medizinethische Entscheidungen sind, sondern auf einem besonderen Lebenstil beruhen $[\ldots]$ «.

3 Andreas Frewer/Christof Müller-Busch/Daniel Schäfer, Neue Kunst des Sterbens? Zur Kultur der Medizin am Lebensende, in: Perspektiven zum Sterben. Auf dem Weg zu einer Ars moriendi nova?, hg. v. dens., Stuttgart 2012, 9-14, hier 12.
} 
zukünftigen Ablebens $[\ldots]$ gestalten und verbessern ${ }^{4}$ und eine neue Sterbekultur etablieren zu wollen.

Dass der Ruf nach einer neuen ars moriendi ertönt, verdankt sich einer Reihe von Gründen. Allen voran steht die Gewissheit, dass die meisten von uns mehr oder weniger langwierige Sterbeprozesse zu gewärtigen haben und sich der Wunsch nach einem raschen Tod für kaum jemanden erfüllen wird. Weniger als fünf Prozent der Menschen sterben einen Sekundentod, über die Hälfte lebt zwei bis drei Jahre mit einer schweren Krankheit, und 30 bis 40 Prozent werden zukünftig mehrere Jahre an Demenz leiden, bevor sie sterben. ${ }^{5}$ Mit Blick auf solche Prognosen drängt es sich regelrecht auf zu fragen, wie mit dem Sterben - im Sinne einer letzten Lebensphase bei schwerer Krankheit mit Pflegebedürftigkeit und längerem Siechtum - umgegangen werden kann und sollte. Das Bemühen um eine gesellschaftlich konsensfähige Sterbekunst erklärt sich aber auch aus der "Unzufriedenheit mit der einseitigen Ethisierung und Moralisierung des Sterbens ${ }^{6}$ und dem daraus entspringenden Wunsch, die als zermürbend erlebten Debatten um Sterbehilfe zu unterlaufen und sich jenseits dieser Zerwürfnisse auf ein gemeinsames Sterbeethos zu einigen. ${ }^{7}$ Bisweilen wird auch die Hoffnung formuliert, eine neue Sterbekunst könne verhindern, dass Menschen "aus Angst vor einem unschönen Tod aktive Sterbehilfe einfordern « ${ }^{8}$. Zudem beklagen die Apologeten einer neuen ars moriendi eine sindividuelle und soziale Thanatophobie «" die offene Gespräche über das Sterben verhindere und damit verunmögliche, unerwünschten Sterbeverläufen vorzubeugen.

Wer heute eine ars moriendi nova postuliert, hat meist nicht im Sinn, diese für Christinnen und Christen zu reservieren, sondern sie Menschen jedweden Glaubens (oder Unglaubens) angedeihen zu lassen. Damit sie in einer säkularen Welt bestehen, kommen die

\footnotetext{
4 Daniel Schäfer/Andreas Frewer/Christof Müller-Busch, Ars moriendi nova. Überlegungen zu einer neuen Sterbekultur, in: Perspektiven zum Sterben. Auf dem Weg zu einer Ars moriendi nova?, hg. v. dens., Stuttgart 2012, 13-23, hier 15.

5 Vgl. Gian Domenico Borasio, Über das Sterben. Was wir wissen. Was wir tun können. Wie wir uns darauf einstellen, München 2011, hier 29.

6 Jean-Pierre Wils, Gibt es eine "ars moriendi nova«?, in: Bioethica Forum 7 (2014), 131-136, hier 131.

7 Vgl. Illhardt, Ars moriendi, 171; Schäfer et al., Ars moriendi nova, 18.

8 Friedemann Nauck, Ars moriendi heute? Neue Sterbekultur aus Sicht der Palliativmedizin, in: Perspektiven zum Sterben. Auf dem Weg zu einer Ars moriendi nova?, hg. v. Daniel Schäfer/Christof Müller-Busch/Andreas Frewer, Stuttgart 2012, 155-158, hier 157.

9 Schäfer et al., Ars moriendi nova, 18.
} 
Vorschläge für eine gegenwärtige Sterbekunst deshalb häufig weltanschaulich neutral daher. Gleichwohl soll der Bezug zur spätmittelalterlichen ars moriendi lebendig bleiben, ohne dass deswegen jedoch eine "idealisierte, in die Vergangenheit projizierte und ästhetisierte Sterbekunst « ${ }^{10}$ propagiert und eine historisch situierte ars moriendi unkritisch für die Gegenwart adaptiert würde. Was aber bietet eine neue ars moriendi, die aus früheren Zeiten mehr als das blosse Etikett erbt? Dieser Frage gehe ich im Folgenden nach. Dem Blick auf die spätmittelalterliche ars moriendi schliessen sich die Darstellung zweier Vorschläge für eine gegenwärtige Sterbekunst und deren kritische Diskussion an. Als zentral kristallisiert sich dabei eine Frage heraus: Wenn der christliche Glaube an ein Weiterleben der Seele nach dem Tod wegfällt, Zweck der traditionellen Sterbekunst aber war, die Rettung der Seele zu befördern, was tritt in der ars moriendi nova an diese Leerstelle? Ich werde die These entfalten, dass der Wunsch nach Erlösung bereits im Diesseits nach Erfüllung ruft - mit der anforderungsreichen Folge, das Sterben optimal gestalten und die eigene Seele selbst zurVollendung führen zu müssen.

\section{Ars moriendi im Spätmittelalter}

Zwischen dem 15. und 18. Jahrhundert etablierte sich in Europa die Gattung der christlichen ars moriendi-Literatur, die praktische Hilfe bei der Vorbereitung auf den Tod versprach und sich enormer Beliebtheit erfreute. Diese "Textgruppe der spätmittelalterlichen Erbauungsliteratur ${ }^{11}$ begegnete der Furcht vor ewiger Verdammnis und leitete dazu an, die Seele auf den Tod und damit auf die Begegnung mit ihrem Schöpfer vorzubereiten, getragen von der Annahme, dass der Einzelne einen Beitrag zu seinem Seelenheil leisten konnte. Kurz gesagt, bot sie Rat, wie sich die Chancen auf den Himmel erhöhen und die Verweildauer im Fegefeuer verkürzen liesse. ${ }^{12}$ Massgeblich stiess die Entwicklung der neuen Textgattung der französische Theologe Jean Gerson an, dessen 1408 erschienenes Opus tripartitum im dritten Teil von der Sterbekunst, De arte

\footnotetext{
${ }^{10}$ Ebd., 15.

11 Wolfgang Uwe Eckart,Verlorene Kunst. Altersgebrechen angesichts des Todes und ars moriendi aus kulturhistorischer Perspektive, in: Handbuch Sterben und Menschenwürde, hg. v. Michael Anderheiden/Wolfgang Uwe Eckart, Berlin 2012, 51-70, hier 60 .

12 Kate Thornton/Christine B. Philips, Performing the good death: the medieval ars moriendi and contemporary doctors, in: Medical Humanities 35 (2009), 94-97, hier 95.
} 
moriendi, handelt und heute als "prototypisch für die Textgruppe « ${ }^{13}$ der ars moriendi-Literatur gilt. Gersons Werk umfasst Mahnungen zum Vertrauen auf Gott, Fragen nach dem Glauben des Sterbenden, Gebete und Anweisungen für den Seelsorger am Sterbebett. ${ }^{14}$ Während sich das lateinische Opus tripartitum vornehmlich an Priester richtete, entstand daneben eine volkssprachliche Sterbeliteratur für Laien. Deren bekanntestes Beispiel ist die auch für Analphabeten verständliche "Bilder-Ars", die lebhaft veranschaulichte, was den Menschen im Sterben erwartete. In je fünf Holzschnitten zeigte das Mitte des 15. Jahrhundert entstandene Werk, wie der Teufel und die Engel Gottes in der Todesstunde um die Seele des Sterbenden kämpfen. Während der Teufel den Menschen mit den fünf Anfechtungen des Unglaubens, der Verzweiflung, der Ungeduld, des Hochmuts und der Habsucht quält, ermutigen ihn die Engel, dem Teufel Widerstand zu leisten, und zwar durch Glauben, Hoffnung, Geduld, Demut und Freigiebigkeit. ${ }^{15}$ In ähnlicher Weise widmeten sich auch andere Werke der ars moriendi-Literatur den Ereignissen im Sterbebett, etwa das im 15. und 16. Jahrhundert zu einem Beststeller in Europa avancierte Speculum artis bene moriendi (auch: Tractatus artis bene moriendi). Typischerweise enthielt ein damaliges Sterbebuch Ermahnungen zum Gottvertrauen und zur Orientierung am Vorbild des Sterbens Jesu Christi, Fragen zum Glauben, Gebete um Sündenvergebung, Ratschläge zum Widerstand gegen die teuflischen Versuchungen und Anfechtungen im Sterbebett und Anweisungen an die Seelsorger. ${ }^{16}$

Der Tod war im Mittelalter allgegenwärtig. Kriege, Hungersnöte und Seuchen, allen voran die Pest, stellten ständige Bedrohungen dar und nährten die Furcht vor einem plötzlichen Tod. Unvorbereitet zu sterben, galt als Gefahr, weil von der sittlichen Verfassung in der Sterbestunde abhing, was den Menschen nach dem Tod blühte: ewiges Heil oder ewige Verdammnis. ${ }^{17}$ Damit konzentrierte sich alles auf die Todesstunde als den Moment letzter und unwiderruflicher

\footnotetext{
${ }^{13}$ Eckhart,Verlorene Kunst, 61.

${ }^{14}$ Vgl. Austra Reinis, Ars moriendi - Ritual- und Textgeschichte, in: Sterben und Tod. Ein interdisziplinäres Handbuch, hg. v. Héctor Wittwer/Daniel Schäfer/Andreas Frewer, Stuttgart 2010, 159-164, hier 159.

15 Vgl. ebd., $159 f$.

${ }^{16} \mathrm{Vgl}$.Austra Reinis, Die lutherische »ars moriendi« im ausgehenden 16. Jahrhundert am Beispiel der Tätigkeit des Theologen und Pfarrers Ägidius Hunnius (1550-1603), in: Sterbeprozesse - Annäherungen an den Tod, hg. v. Michael Rosentreter/Dominik Groß/Stephanie Kaiser, Kassel 2010, 51-64, hier 52.

${ }_{17}$ Vgl. Rainer Rudolf, Ars moriendi: Mittelalter, in: TRE, Bd. 4, Berlin/New York 1979, 143-149, hier 144.
} 
Entscheidung. Wenn die Seele im Augenblick des Ablebens nicht bereit war, der Mensch seine Sünden nicht gebeichtet hatte oder im Todeskampf den Einflüsterungen des Teufels erlegen war, bestand keine Hoffnung auf Rettung.

Das hatte mehrere Konsequenzen. Gerade angesichts der ständigen Bedrohung durch Seuchen und andere Infektionskrankheiten musste man sein Leben stets tugendhaft und gottgefällig gestalten, um nicht im falschen Moment vom Tod überrascht zu werden. Die alltägliche Lebensführung war damit geprägt vom Gedanken an den Tod, die Sterbevorbereitung begann mitten im Leben. "Media vita in morte sumus«: Die mittelalterliche Wendung gemahnt, diesen Umstand nicht zu vergessen und stets gefasst zu sein auf den Tod.

Die letzte Lebensphase erhielt aber dennoch besonderes Gewicht. Denn im Sterbebett drohten dem Menschen letzte Anfechtungen, denen er widerstehen musste. Der Teufel lockte ihn mit seinen Versuchungen, so dass das Ringen und der Kampf ums eigene Seelenheil bis zum letzten Atemzug währten und die Todesstunde zur Bewährungsprobe der sittlichen und spirituellen Festigkeit wurde. Wer vor dem Ableben alle seine Sünden einem Priester beichtete und sich zudem den teuflischen Einflüsterungen im Sterbebett widersetzte, durfte zwar auf die Rettung seiner Seele hoffen, doch sicher sein konnte er sich nicht, ob er den Stand der Gnade erlangt hatte und zu den Erwählten gehörte. Die Entscheidung über Rettung oder Verdammnis blieb Gott anheim gestellt, und ein moralisch einwandfreies Leben war nur eine notwendige, nicht aber hinreichende Bedingung für das Heil. ${ }^{18}$ Nach dem Tod folgte die Reinigung der Seele im Fegefeuer. ${ }^{19}$

Die ars moriendi-Literatur widmete sich entsprechend nicht allein der unmittelbaren Todesvorbereitung im Sterbebett, sondern auch der ars vivendi, der rechten Lebensführung, und lässt sich als in die "christliche Moral-Diätetik des Lebens [...] inkorporierte Kunst des Sterbens $\aleph^{20}$ verstehen. Waren die frühen Abhandlungen über die Sterbekunst als Handreichungen für die priesterliche Seelsorge am Sterbebett gedacht, so bereiteten sich mit der in die Volkssprachen übersetzten ars moriendi-Literatur auch Laien auf ihr eigenes Sterben vor oder standen gemäss der Anleitungen in den Sterbebüchern ihren Mitmenschen auf dem Sterbebett bei, wenn kein Priester verfügbar war. ${ }^{21}$ Denn konnten in den mittelalterlichen Krisenzeiten

${ }^{18}$ Vgl. Reinis, Ars moriendi, hier 159.

${ }^{19}$ Vgl. Reinis, Die lutherische »ars moriendi«, 52.

${ }^{20}$ Eckart,Verlorene Kunst, 60.

${ }^{21}$ Vgl. Reinis, Die lutherische "ars moriendi«, 52. 
Ärzte nicht mehr helfen und mangelte es an Priestern, so übernahmen Laien die Aufgabe der Sterbebegleitung.

\section{Ars moriendi heute}

Heutige Ansätze einer ars moriendi kommen darin überein, dieVorbereitung auf das Sterben anzumahnen und es selbst als "performance ${ }^{22}$ zu betrachten, die es zu bestehen und gestalten gilt. Ich greife zwei Beispiele von Konzeptionen einer ars moriendi nova heraus, die sich beide als weltanschaulich neutral verstehen, wobei die erste Variante sich nur der Methode, nicht aber dem Inhalt nach an der spätmittelalterlichen Sterbekunst orientieren will, während sich die zweite Version auch inhaltlich an der früheren ars moriendi orientiert.

Schäfer und Kollegen ${ }^{23}$ erklären zum Ziel einer Wiederbelebung der ars moriendi, die verfahrene Debatte über Sterbehilfe neu auszurichten. Ihnen schwebt unter dem Schlagwort ars moriendi eine Sterbeberatung mit hohem praktischen Nutzen vor, eine Anleitung zum guten Sterben, die Hinweise auf mentale, visuelle, pädagogische und pragmatische Aspekte der Vorbereitung auf den Tod umfasst. Die ersten beiden Punkte benennen die Art und Weise, wie jeder Einzelne sich auf sein Sterben einstellen kann: Mental soll er / sie sich wappnen, indem er sich nicht nur seine Endlichkeit bewusst macht, sondern auch möglichst viele Informationen über heute typische Sterbeverläufe sammelt. Er / sie soll wissen, dass die meisten Menschen einen längeren Sterbeprozess durchlaufen, sich im Alter und bei Krankheit die eigene Einstellung wandeln kann und Lebensumstände womöglich als erträglich erlebt werden, die zuvor, in gesundem Zustand, den Wunsch nach einem raschen Tod weckten. Dem entspricht in der spätmittelalterlichen ars moriendiLiteratur, über die drohenden Anfechtungen im Sterben, über den Sinn des Todes und das Schicksal der Seele aufzuklären. Zur visuellen Sterbevorbereitung dient die Imagination unterschiedlicher Sterbeweisen mit dem Ziel, das Sterben zu akzeptieren, ebenso wie einst die Bilder-Ars dabei half, sich die Sterbestunde anschaulich vorzustellen. Als Sterbepädagogik soll die neue ars moriendi Eingang in den Schulunterricht finden und im Selbststudium angeeignet werden, anknüpfend an den Gedanken der traditionellen ars moriendi, dass sich das Sterben lernen lässt wie ein Handwerk. Und pragmatisch wird sie, wo sie konkrete Hilfestellung leistet und das Gespräch

22 Thornton/Philips, Performing the good death, 95 .

${ }^{23}$ Schäfer et al., Ars moriendi nova, 18-21. 
über das Sterben eröffnet, heute etwa in Talkshows, einst, indem die ars moriendi-Literatur Priester in der Sterbeseelsorge unterwies und Laien Ratschläge erteilte. Zwar mahnen die Autoren auch die Verbesserung der Begleitung Sterbender an, primär findet ihre Variante einer ars moriendi nova jedoch nicht im Sterbeprozess oder gar in der Todesstunde Anwendung, sondern bedeutet eine Auseinandersetzung mit Tod und Sterben möglichst bereits zur Schulzeit und dann kontinuierlich im weiteren Leben. Dabei scheint der Grundgedanke zu sein, dass es sich leichter stirbt, wenn eine Person bereits sämtliche Eventualitäten und Unwägbarkeiten eines Sterbeprozesses frühzeitig kontempliert und sich mit dem Tod vertraut gemacht hat.

Anders verfährt Carlo Leget, dessen Variante einer ars moriendi nova sich eng am traditionellen Vorbild orientiert und für die unmittelbare Anwendung im Rahmen der Spiritual Care konzipiert ist. ${ }^{24}$ Die fünf Kampfgründe zwischen teuflischen Anfechtungen und himmlischen Tröstungen ersetzt er durch fünf moderne Spannungsfelder, mit denen er heutige Sterbende konfrontiert sieht und die den spätmittelalterlichen Vorläufern entsprechen sollen: Autonomie, Schmerzkontrolle, Bindung an Weltliches, der Rückblick auf das eigene Leben sowie Glaubensfragen. In Legets Konzeption der Sterbekunst streiten sich nicht mehr Engel und Teufel, Gut und Böse, doch stehen dem Sterbenden nach wie vor zwei extreme Möglichkeiten offen, mit den verschiedenen Dimensionen seines Sterbens umzugehen. Wo einst der Teufel an den Stolz des Menschen appellierte, niemanden über sich herrschen zu lassen, auch Gott nicht, kann heutige Sterbende ihr Streben nach Autonomie zum Unguten führen - dann nämlich, wenn sie nicht mehr wahrnehmen, stets mit anderen Menschen in Beziehungen verbunden zu sein. Anders als die spätmittelalterliche ars moriendi schlägt Leget nun nicht vor, das Gegenteil zu tun, also die Autonomie ganz fahren und andere Menschen für sich entscheiden zu lassen, sondern einen Mittelweg zwischen den beiden Extremen zu wählen. Ähnliches schwebt ihm auch für die übrigen vier Gegensatzpaare vor. Eine Balance zwischen Ungeduld und Geduld muss der Sterbende bei der Kontrolle seiner Schmerzen finden und die Anhänglichkeit an weltliche Güter gegenüber dem Loslassen alles Irdischen abwägen, so wie sich der einstige Sterbende der Habsucht erwehren musste. Verzweiflung mag sich beim Rückblick auf das eigene Leben breitmachen, der damit begegnet werden kann, sich zu versöhnen, oder aber, wo das nicht möglich ist, die Dinge auf sich beruhen zu lassen. Und bedrohte einst der Unglauben als schlimmste Anfechtung den Sterben-

\footnotetext{
${ }^{24}$ Leget, Retrieving the ars moriendi tradition.
} 
den, so begleiten womöglich auch heute quälende Glaubens- und Sinnfragen den Sterbeprozess, die sich lösen lassen sollen, wenn er zwischen einer Sinnsuche im Glauben und dem Vertrauen auf sein Wissen den Ausgleich findet. In allen fünf Bereichen plädiert Leget dafur, in einem Reflexionsprozess zu einer individuell stimmigen Balance zwischen den Extremen zu gelangen.

Legets Modell einer ars moriendi nova wird im Rahmen der Spiritual Care am Universitätsspital Louvain in die Praxis umgesetzt. Konkret bedeutet das, dass alle an der Versorgung eines Sterbenden beteiligten Personen in den Patientenakten unter den fünf Stichpunkten festhalten, was den Patienten bewegt, und dies als Grundlage für den interprofessionellen Dialog nutzen. Die ars moriendi wird damit zu einer Angelegenheit der Palliative Care und verwirklicht sich, anders als der Ansatz von Schäfer und Kollegen, im Sterbeprozess.

\section{Sterbeoptimierung als Seelenrettung}

Von ihren Ursprüngen hat sich die heutige ars moriendi weit entfernt. Bei beiden vorgestellten Ansätzen ist spürbar, dass sie darauf verzichten wollen festzulegen, wie ein Sterbeprozess im Detail abzulaufen habe. Die Ratschläge beziehen sich auf die Form, mit dem Sterben umzugehen, was vor allem bewusst und reflektiert erfolgen soll, statt zu einem bestimmten Verhalten im Leben wie im Sterben aufzufordern, wie es die spätmittelalterliche ars moriendi vorsah. Ob jemandem im Sterben Glauben, Spiritualität und Religion helfen, bleibt in den skizzierten Varianten einer ars moriendi nova dem Einzelnen überlassen und soll nicht Bestandteil einer kollektiv geteilten Sterbekunst sein. Das religiöse Bekenntnis wandelt sich in ein "Bekenntnis zur eigenen Biografie und den darin gelebten Wert- und Glaubensvorstellungen ${ }^{25}$. Damit macht sich die weltanschaulich neutrale ars moriendi nova zwar den historisch geprägten Namen zu Eigen, doch werden zentrale Momente der spätmittelalterlichen Sterbekunst negiert, vor allem die Perspektive auf das Jenseits und die Verwurzelung in einer bestimmten religiösen Tradition.

Wird jeglicher Jenseitsglaube zwar nicht ausgeschlossen, aber doch ausgeklammert, verzichtet man allerdings nicht bloss auf einen Aspekt der traditionellen ars moriendi, sondern auf ihr Fundament, nimmt also eine grundlegende Umdeutung vor. Ihre Bedeutung

${ }^{25}$ Traugott Roser, Ars moriendi nova aus Sicht von Theologie und Spiritual Care, in: Perspektiven zum Sterben. Auf dem Weg zu einer Ars moriendi nova?, hg. v. Daniel Schäfer/Christof Müller-Busch/Andreas Frewer, Stuttgart 2012, 171-179, hier 173. 
gewann die frühere Sterbekunst ausschliesslich aus der Sorge um das Schicksal der Seele nach dem Tod. Nichts weniger als das ewige Heil stand in der Sterbestunde auf dem Spiel. Sämtliche Ratschläge in der ars moriendi-Literatur richteten sich allein darauf, den Menschen auf den finalen Kampf zwischen Teufel und Engeln um seine Seele und auf sein eigenes Zutun für einen glücklichen Ausgang vorzubereiten. Was aber sind die Folgen, wenn aus der ars moriendi eine Sterbekunst ohne Jenseitsperspektive wird? Was tritt an die Stelle der Rettung der Seele?

Fehlt die Orientierung auf ein Jenseits hin, weil der Glaube an ein Leben nach dem Tod oder zumindest die Angst vor der Hölle geschwunden sind, so bleibt eine rein diesseitige Praxis übrig, deren Sinn darin besteht, den Sterbeprozess so angenehm oder wenig quälend wie möglich zu gestalten, wie bei Schäfer et al. deutlich wurde: Die zeitlebens währende Auseinandersetzung mit dem Sterben soll wohlinformierte Sterbende hervorbringen, die ihr nahendes Lebensende kaum mehr schreckt, weil sie sich so gut als möglich für sämtliche Eventualitäten gewappnet haben. Gefahr droht allenfalls vom Sterbeprozess selbst, der körperliche Schmerzen und seelisches Leiden bedeutet und deswegen auch heute noch zu Kämpfen in verschiedenen Dimensionen führen kann, wie Leget meint. Dabei verlagert sich der Streit zwischen Teufel und Engeln als von aussen kommenden Streitern um die menschliche Seele in einer Welt ohne Jenseitsbezug ins Innere des Menschen, bleibt aber bis zuletzt nicht weniger aufreibend. Auch hier geht es um mehr als bloss die letzten Tage und Stunden eines Sterbeprozesses.

Denn die Sorge um die Seele steht ebenso im Mittelpunkt der ars moriendi nova. Wie in beiden zeitgenössischen ars moriendi-Konzeptionen deutlich wurde, mündet dies nun allerdings in der Mahnung, darauf hinzuarbeiten, als reife und mit sich selbst versöhnte Persönlichkeit zu sterben, die nicht mehr mit dem Tod hadert, sondern zu einer gelassenen Haltung und innerem Frieden findet, weil sie sich rechtzeitig auf das Sterben vorbereitet hat. Heil oder Verdammnis warten nicht mehr im Jenseits, sondern bereits im Hier und Jetzt muss sich entscheiden, wie es um die Seele steht, ob der Reifeprozess vollendet gelingt und am Schluss ein "reales Ja zum Sterben ${ }^{26}$ steht. Auch wenn sich die "säkularisierten Paradiesesziele ${ }^{27}$ allein im Diesseits verwirklichen lassen, bleibt die Unwiderruflichkeit des

\footnotetext{
${ }^{26}$ Schäfer et al., Ars moriendi nova, 20.

${ }^{27}$ Dietrich von Engelhardt, Lebenskunst (ars vivendi): Kunst des Krankseins (ars aegrotandi) und Kunst des Sterbens (ars moriendi), in: Zeitschrift für medizinische Ethik 52 (2006), 239-248, hier 243.
} 
Entscheids zwischen Wohl und Wehe: Entweder gelangt die Seele zur Erlösung, bevor sie im Tod verschwindet - oder sie wird es nie mehr.Wie einst, geht es also auch heute noch um alles, um der Seele Heil oder Unheil.

Gerade weil die gegenwärtige ars moriendi die Erfüllung zu Lebzeiten sucht, kommt auch hier der Sterbephase hohe Bedeutung $\mathrm{zu}$, denn nun besteht die letzte Chance, Unabgeschlossenes zu vollenden oder aber sich damit auszusöhnen. Nicht mehr moralisch einwandfrei und seiner Sünden in der Beichte entledigt, um vor Gottes Urteil zu bestehen, muss der Sterbende in seine Todesstunde eingehen, aber ebenso geläutert und innerlich gereift, indem er sich mit sich selbst und den Seinen versöhnt hat und alle Beteiligten einander wechselseitig Vergebung zugesprochen haben. Das Aufspüren und Bekennen der Sünden, das zur früheren ars moriendi gehörte, verwandelt sich in eine nicht minder anforderungsreiche introspektive Technik der Selbstformung. Das gute Sterben zu bewerkstelligen, erweist sich dabei auch gegenwärtig als "moral activity ${ }^{28}$, insofern Gelassenheit, persönliche Reife und die Versöhnung mit sich selbst und mit anderen als innere Werte zum Ziele stehen. Dabei findet jedoch nachgerade eine Umkehrung des spätmittelalterlichen Verständnisses statt: Prägten die damalige Sterbekunst "Moraldefizite ${ }^{29}$, die es auszugleichen galt, verkehrt sich die Defizitorientierung nun in einen unbedingten Optimierungswillen, der aus dem Sterben eine nicht minder anforderungsreiche Angelegenheit macht, als es in der Vergangenheit der Fall war. Der Sterbende muss sich nun nicht mehr bemühen, eigene Mängel auszugleichen, um zuletzt möglichst mit einer positiven moralischen Bilanz vor Gott zu treten, der ihn erretten und in den Himmel aufnehmen soll.Vielmehr muss er das Seelenheil eigenständig zustande bringen, stets den gesunden und individuell passenden Mittelweg in seinen Haltungen und Entscheidungen wählen und dank jahrzehntelanger Vorbereitung den Widrigkeiten des Sterbeprozesses seelenruhig trotzen. Die Sterbeoptimierung der ars moriendi nova reicht, so bei Schäfer und Kollegen, weit in das Leben herein und kommt in der Sterbephase zu ihrem Höhepunkt. Dass ars moriendi als "Sterbekunst" übersetzt und nicht, wie es dem lateinischen Wort entspräche, als Handwerk verstanden wird, verschärft den Eindruck, dass es sich um eine perfektionierbare Fertigkeit handele und das Sterben zu einer »künstlerischen

${ }^{28}$ Thornton/Philips, Performing the good death, 94.

29 Wils, ars moriendi, 24. 
Performance « ${ }^{30}$ werden könne. Eine solche Ästhetisierung verklärt das Sterben in einer Weise, die mit mühevollen oder von Verzweiflung begleiteten Sterbeprozessen wenig zu tun hat. Das lateinische "ars" hingegen bezeichnet die Fertigkeit eines Handwerkers - und damit ein vergleichsweise nüchternes Können. ${ }^{31}$

Nicht hinterfragt werden dabei die normativen Vorannahmen: Weshalb soll es gut sein, sich mit dem Tod zu versöhnen und seinem Lebensende gelassen entgegen zu sehen? Stirbt tatsächlich besser, wer frühzeitig an den eigenen Tod denkt? Verbessert es das Sterben, wenn sich der Tod tabulos immer und überall thematisieren lässt? Die Verfechterinnen und Verfechter einer ars moriendi nova verfolgen, ohne es klar zu benennen, ein durchaus fragwürdiges Sterbeideal. Indem sie das Sterben als gestalterische Optimierungsaufgabe konzipieren, die der Einzelne zu leisten und der er sich schon früh im Leben zuzuwenden hat, bleiben andere Umgangsformen mit dem Lebensende nicht bloss unterbelichtet, sondern werden indirekt auch mit einem Makel versehen, entsprechen sie doch nicht dem state of the art des Sterbens. Sich der Sterbeoptimierung zu entziehen und den Tod bis zuletzt zu verdrängen, keine Vorkehrungen zu treffen und auch im Sterbeprozess kein Programm inneren Wachstums durchlaufen zu wollen, ist in der ars moriendi nova schlicht nicht vorgesehen. Dass die Pointe der traditionellen ars moriendi, nämlich die Rettung der Seele nicht durch eigene Anstrengungen im Diesseits zu bewerkstelligen, sondern sich zwar auf die Begegnung mit Gott so gut wie möglich vorzubereiten, die Vollendung aber erst - und auch nur im besten Fall - im Jenseits zu erwarten, in der gegenwärtigen Sterbekunst ignoriert wird, hat zur paradoxen Folge, dass unter dem Mantel weltanschaulicher Neutralität neue Normierungen des Sterbens eingeführt werden, die weit anspruchsvoller sind als frühere, eben weil die letzte Chance auf Erfüllung nur im Hier und Jetzt besteht.

- Nina Streeck ist Assistentin an der Professur für Spiritual Care und Doktorandin am Institut für Biomedizinische Ethik und Medizingeschichte an der Universität Zürich.

${ }^{30}$ Reiner Sörries, Der Tod ist der Feind des Lebens, oder:Vom Irrtum, das Sterben könne eine Kunst sein, in: Perspektiven zum Sterben. Auf dem Weg zu einer Ars moriendi nova?, hg. v. Daniel Schäfer/Christof Müller-Busch/Andreas Frewer, Stuttgart 2012, 159-162, hier 160.

${ }^{31}$ Vgl. Wils, ars moriendi, 25. 